\title{
Diagnosis of Inclusion
}

\author{
Kristin M. Ikeda, Sumit Das, Michael Strong, Seyed M. Mirsattari, Andrew Leung, David Steven, \\ Robert Hammond
}

Keywords: Amyotrophic lateral sclerosis, CNS inflammation, infections of the nervous system, immune system, neurology adult, neuropathology

doi:10.1017/cjn.2015.2

Can J Neurol Sci. 2015; 42: 138-143

\section{Case Presentation: Dr. IKeda}

\section{History}

An 83-year-old, right-handed female was referred for progressive weakness. Her medical history included hypertension, hypercholesterolemia, gastroesophageal reflux, and bilateral cataract removals. Her medications included pregabalin, trazodone, olmesartan, amlodipine, escitalopram, pantoprazole, aspirin, vitamins, and stool softeners.

Her initial symptoms began four months before admission when she noticed her right leg was dragging when walking. Her weakness was progressive and began to involve the left leg two months after onset. Three months after onset, she was unable to ambulate and also had progressive left arm weakness. At the time of admission, four months after onset, she was unable to move both legs or her left arm.

There were no significant fluctuations in her symptoms. She denied visual symptoms, facial weakness, paresthesias, swallowing difficulties, or changes in her voice. She did not have any sensory changes. The patient and family did not endorse any cognitive difficulties. Systemic review of systems was unremarkable.

\section{Physical Examination}

Her systemic examinations_-including cardiovascular, respiratory, abdominal, and dermatological—were unremarkable. Her mental status and language examinations were normal. A Montreal Cognitive Assessment score was 25/30 (points lost for attention, 1; language, 1 ; and delayed recall, 3). Her cranial nerve examination was significant for a power of $4 / 5$ in the sternocleidomastoids bilaterally and $0 / 5$ in her left trapezius. The remainder of her cranial nerves was normal.

On motor examination, she had spastic tone in all four extremities and she had two to four beats of clonus at the ankles. Power was $0 / 5$ throughout the left upper extremity and both lower extremities. She had full power in the right arm with the exception of shoulder abduction, which was $4 / 5$. She was diffusely hyperreflexic with a crossed adductor on the right and bilateral extensor plantar responses.

Sensory examination revealed normal sensation to pinprick, but elevated threshold to vibration at both distal interphalangeal joints in the fingers, the right great toe, and decreased vibration sense to the left ankle. She had extinction to double-simultaneous stimuli on the left arm and leg.

\section{Investigations Available at the Time of Consultation}

Routine bloodwork including complete blood count, electrolytes, liver enzymes, uric acid, creatinine, and thyroid-stimulating hormone was normal. Vitamin B12 level was borderline low at $122 \mathrm{pmol} / \mathrm{L}$ (lower limit, $107 \mathrm{pmol} / \mathrm{L}$ ). A fasting glucose was $6.2 \mathrm{mmol} / \mathrm{L}$, and a cholesterol profile showed total cholesterol $5.24 \mathrm{mmol} / \mathrm{L}$, triglycerides $1.12 \mathrm{mmol} / \mathrm{L}$, low-density lipoprotein $3.45 \mathrm{mmol} / \mathrm{L}$, and high-density lipoprotein $1.27 \mathrm{mmol} / \mathrm{L}$ (total cholesterol to high-density lipoprotein ratio 4.12 .

The patient had a CT scan of the head with and without contrast with a small area of decreased attenuation at the gray-white matter junction in the right parietal lobe consistent with an area of chronic ischemia. An MRI of her spine revealed multilevel degenerative disc disease with moderate spinal stenosis and foraminal narrowing at L3-4 and L4-5.

Electromyography and nerve conduction studies had been performed one month before evaluation and were significant for $1+$ fasciculations in the right rectus femoris muscle. The remainder of the studies was normal.

\section{Initial Discussion: Dr. Strong}

The presentation is that of an 83-year-old, right-handed lady with a rapidly progressive motor system dysfunction marked initially by weakness in the right lower limb, followed thereafter by left lower limb and then the left arm. This progresses over the course of four months to the point where the patient is essentially functionless in the lower limbs, with profound weakness in the left upper limb while sparing the right upper limb, with the exception of shoulder abduction. There is also sparing of bowel and bladder function. The sensory extinction is the only evidence of non-motor pathology and, for reasons which I will discuss, could be considered as independent of a pure motor syndrome.

Although there are several ways to discuss this case, the initial step should be an attempt to localize the pure motor system dysfunction. The rapidly progressive paralysis beginning first in one leg, moving to the next, and then to the ipsilateral arm

From the Departments of Clinical Neurological Sciences (KMI, MS, SMM, AL, DS, RH); Pathology, Division of Neuropathology (SD, RH); Diagnostic Imaging, Division of

Neuroradiology (AL), London Health Sciences Centre, London, Ontario, Canada Received June 16, 2014. Final Revisions Submitted December 23, 2014. Correspondence to: Robert Hammond, Rm. A-148, Department of Pathology, LHSC-UH, 339 Windermere Road, London, ON N6A 5A5, Email: robert.hammond@lhsc.on.ca 
might be suggestive of pathology at the level of the foramen magnum. However, the pattern is wrong: motor deficits are often associated with sensory dysfunction in one arm, then progress to the ipsilateral leg followed by the contralateral leg, ultimately insidiously presenting as a spastic quadriparesis in association with lower cranial nerve deficits such as tongue wasting and fasciculations and sternomastoid weakness. At this age, one would consider degenerative disease at the level of $\mathrm{C} 1$ or the odontoid process and include meningioma (more typical in middle-aged women) as well as rheumatoid pannus formation with degeneration. However, to progress to the point of profound lower limb weakness without bowel or bladder dysfunction is distinctly unusual. The same logic would apply for less common cervical extra-axial pathology. In this sense, the weakness of the sternomastoids is important and would move the localization away from the foramen magnum and $\mathrm{C} 1$ to approximately $\mathrm{C} 2$ or C3. The weakness of shoulder abduction on the right and flaccid left upper limb would suggest a level of at least C4 or C5. This would suggest a mid- to high cervical localization and, given the pure motor manifestation, involvement of both the ventral spinal cord as well as the corticospinal tracts. The highly asymmetric nature of this and the sparing of bowel and bladder dysfunction is quite atypical.

Given this anatomic localization and recognizing that we have not yet dealt with the sensory dysfunction, the primary site of pathology is likely to be the level of the spinal cord itself. At this point, one needs to consider both intra- and extraaxial pathology sufficient to give rise to predominantly ventral spinal cord syndrome while also involving the corticospinal tracts and sparing the spinothalamic tracts. Vascular pathology would be a prime candidate given the vascular supply to the spinal cord itself, and raises the possibility of an arteriovenous fistula or dural fistula. Similarly, extra-axial compressive pathology either in the form of degenerative disease or as an infiltrative pathology of the vertebral bodies needs to be considered, although the latter would be rapidly discarded because of the absence of pain and the rapid progression. She is too well systemically for this to be an epidural abscess. It will be critical to have adequate neuroimaging of the spinal cord to exclude any of these entities. I would also tend to think this unlikely given the marked sparing of the right upper limb that, given the profound nature of weakness now present throughout both lower limbs and left upper, is distinctly unusual for any extra-axial pathology at this level.

This then leads to intra-axial spinal cord pathology. Before reaching this conclusion, however, one needs to consider whether this could be a rapidly progressive motor neuron disorder, the likes of which I've not seen before. Although this is a tempting diagnosis given the relatively pure motor disease accompanied by spasticity, it would be unusual to have this profound degree of involvement and yet spare the right upper limb. Neither the history nor examination includes atrophy or fasciculations that, given the four-month course, would have been expected by this point. Thus, although I think it unlikely that this is any variant of a pure motor neuron disease or a motor axonal neuropathy, the electrophysiological studies will bear repetition and will be critical.

Having said this, it is still more likely that this is intra-axial spinal cord pathology. At this point, the sensory extinction (localizing to right primary sensory cortex and association areas) and pathology in the right parietal lobe, consistent with subcortical pathology, becomes important to the differential diagnosis. If the two processes are linked, then one must consider a more disseminated white matter or multifocal disease process. Vasculitis, sarcoid, syphilis, and tuberculosis should all be considered within the differential diagnosis, as would a diffuse demyelinating process. However, her age would argue against the latter, as would the absence of previous events. We do not have imaging that would help define the pathology in the right parietal lobe as being vascular, but if it is that at this stage, it would suggest an underlying vasculopathy. Given our proposed localization of the spinal cord pathology and involvement of the anterior spinal artery, which is supplied through the vertebral arteries, it would be reasonable to consider vasculopathy in the differential. The absence of bowel or bladder dysfunction, the insidious nature of the progression, and the absence of pain all mitigate against this being a spinal cord infarction. It does not, however, rule out isolated central nervous system vasculitis- the manifestations of which can be protean. At the same time, one would want to consider immune-mediated processes, including lupus or paraneoplastic disorders; as such, the possibility of an underlying immunocompromised state will have to be considered. Having raised this, and still returning to the mid- to high cervical spinal cord localization, three viral infections should be considered: West Nile virus, Epstein-Barr virus, and cytomegalovirus. Each of these can give rise to a syndrome that can mimic aspects of a transverse myelitis, although each of these would not be expected to have progressed to the extent that this has, and each would be expected to have sensory impairment. Thus, although a spinal fluid analysis will be critical to the differential, I doubt that it will demonstrate an underlying infective process.

At this point, the most parsimonious differential diagnosis rests with two localizations: one to within the ventral spinal cord at the mid- to high cervical level and, second, a lesion involving the cerebral hemispheres, most prominently the right parietal lobe. To link these two processes together, one would consider an inflammatory or immune-mediated process, a disseminated vasculitis, or disseminated white matter disease such as a leukoencephalopathy and potentially sarcoid.

To further the differential diagnosis, I would like to see neuroimaging that includes the high cervical spinal cord with attention to the vascular supply, a spinal fluid analysis, and comprehensive electromyography to ensure that this is not an extremely atypical motor neuron disease.

\section{INVESTIGATIONS AND MANAGEMENT: DR. IKEDA}

Lumbar puncture showed increased protein at $910 \mathrm{mg} / \mathrm{L}$ (reference 200-400) with three nucleated cells. There were no oligoclonal bands present in the cerebrospinal fluid (CSF). Gram stain and culture were negative.

Nerve conduction studies were significant for generalized reduction in motor amplitudes suggesting a pure motor, non-lengthdependent axonal process. There was an incidental left ulnar neuropathy at the elbow. Electromyography demonstrated mild acute denervation in the right tibialis anterior and left vastus lateralis with large motor units recruited in multiple muscles sampled, suggesting a more long-standing neurogenic process, but not confirmatory of any particular diagnosis. Electromyography did not meet criteria for amyotrophic lateral sclerosis. 


\section{NeUroimaging: Dr. LeUng}

MRI of the brain demonstrated subcortical white matter T2 hyperintensity in both frontal and parietal lobes paracentrally, worse on the right (Figure 1). This was mildly expansile. There was similar abnormality in the right subinsular white matter. Following gadolinium administration, there was a small area of enhancement in the left frontal subcortical white matter but no leptomeningeal enhancement. There was no diffusion restriction or hemorrhage. There was no stenosis or filling defect in the dural sinuses. Magnetic resonance angiography was unremarkable. Imaging of the cervical spine was normal, with no evidence of signal change within the cord.

The abnormalities showed progression on the subsequent MRI performed one month later. The subcortical white matter signal change was more extensive and had more mass effect. There were more foci of parenchymal enhancement.

The findings were nonspecific and the differential diagnosis remained broad. Multifocal glioma should be considered. Demyelination was possible, as were infection causing cerebritis and inflammatory conditions such as sarcoidosis. If the patient was immunocompromised, atypical infections and progressive multifocal leukoencephalopathy should be contemplated. The appearance was not typical for vasculitis.

\section{AdDitional InVESTigations: Dr. IKEDA}

Based on the results of the initial investigations, further extensive laboratory investigations were completed to explore potential etiologies of the signal change seen on the brain MRI. Erythrocyte sedimentation rate, C-reactive protein, cytoplasmic antineutrophil cytoplasmic antibodies, perinuclear antineutrophil cytoplasmic antibodies, antiphospholipid antibodies, lupus anticoagulant, rheumatoid factor, C3, C4, anti-double-stranded DNA, and extractable nuclear antigens were negative. Serum and CSF angiotensinconverting enzyme levels were negative. Tests for antinuclear antibodies (ANA) were positive at a titre of 1:2560, and CH50 was $>50$. Rheumatology was consulted for the elevated ANA, who felt this was not significant in this context as there was no evidence of connective tissue disease. They indicated that a positive ANA can be seen in many normal individuals as well as in the context of other inflammatory or autoimmune diseases, infection, and neoplasm.

Infectious workup, including hepatitis B and C serology, arbovirus, Lyme, HIV, and VDRL was negative. Serum protein electrophoresis and tumor markers CA19-9, CEA, and Ca-125 were negative. Routine laboratory investigations including electrolytes urea, creatinine, and liver enzymes were all normal. Complete blood count was normal with a white blood cell count of $4.3 \times 10^{9}$, but a low lymphocyte count of $0.7 \times 10^{9}$ (reference
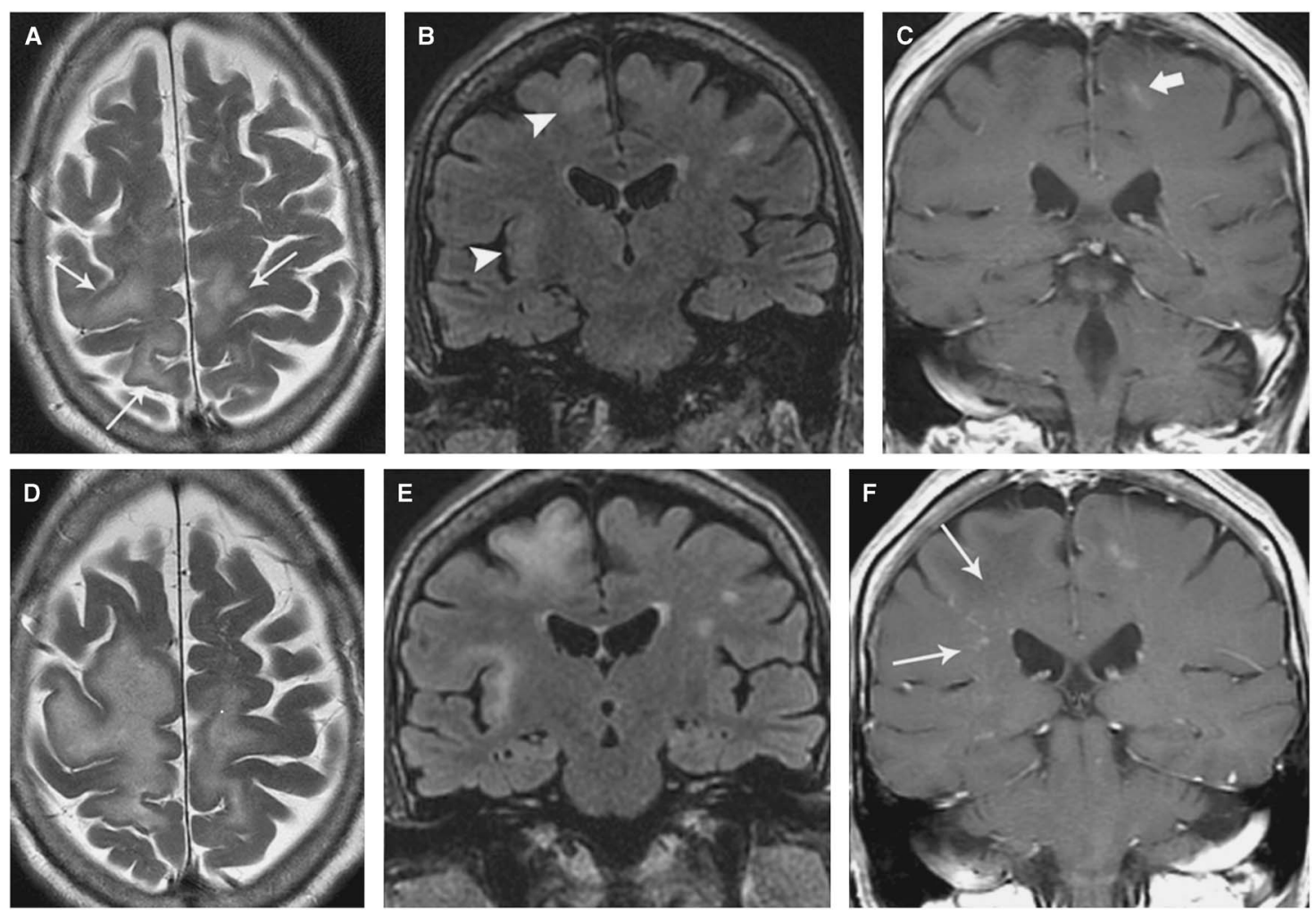

Figure 1: (A) Axial T2 image demonstrates increased parenchymal signal in the frontal and parietal lobes paracentrally, worse on the right. The abnormality is primarily in the subcortical white matter with mild cortical involvement (arrow). Mass effect is minor. (B) A coronal fluid attenuation inversion recovery image shows similar subinsular white matter signal change in the right parietal lobe and insula (arrow heads). (C) Post-gadolinium T1 sequence shows focal enhancement in the left subcortical white matter (short arrow). (D-F) Corresponding images from a subsequent MRI performed one month later show progression. There has been moderate extension of signal change and a mild increase in mass effect. There are new punctate foci of enhancement in the deep white matter on the right (arrows). 
1.5-4.0). Her CD4 count was 0.241 (reference 0.504-1.632) and CD8 count was 0.036 (reference 0.249-0.953).

A CT scan of the chest, abdomen, and pelvis was obtained to rule out a primary neoplasm, which demonstrated pulmonary emboli, but no evidence of neoplasm.

\section{Discussion: Dr. STRONG}

The immunological studies are of some help. Although the positive ANA at 1:2560 is impressive, it is not accompanied by a positive anti-double-stranded DNA and is therefore likely not to be of significance. No other immunological abnormalities are present, although the alteration the CD4/CD8 ratio is intriguing and might suggest an underlying immunocompromised state. The lumbar puncture is helpful in excluding a viral or inflammatory process, with the negative oligoclonal banding also suggesting that this is not multiple sclerosis. The electrophysiological studies confirm that there is no evidence of a disseminated motor neuron disease or an underlying motor axonopathy. Thus, there's no specific reason to include a disseminated motor neuron disease within the differential diagnosis.

The neuroimaging studies help to further define the process. Specifically, there is a disseminated process that is asymmetric with patchy enhancement, and thus consistent with a vascular process such as isolated central nervous system vasculitis, an immunocompromised state with a progressive leukoencephalopathy, or an infectious process. I think it unlikely that this would be accounted for on the basis of a disseminated glioma given the absence of mass effect. Similarly, the previous infective etiologies that were raised would seem unlikely given the negative CSF. At this point, the CT scan of the chest that demonstrated a filling defect in the left main pulmonary artery suggestive of a pulmonary embolism may also suggest an underlying hypercoagulable state and, at this age, a paraneoplastic syndrome. Although magnetic resonance angiogram showed no evidence of an isolated central nervous system vasculitis, this would need to be further pursued through a brain biopsy. To reconcile both the profound motor dysfunction and sensory extinction, a disseminated leukoencephalopathy either as the consequence of a vasculitis, or as a paraneoplastic syndrome, should be considered. The next test should be a brain biopsy.

\section{Neurosurgery: Dr. STEVen}

In light of the imaging and differential diagnosis, we elected to obtain a full-thickness biopsy of cortex and subcortical white matter, including the leptomeninges. A right central craniotomy was performed and a biopsy site chosen in the right frontal lobe, immediately anterior to the Rolandic cortex superiorly. Intraoperative neuronavigation was used to ensure that the specimen was obtained from a region that corresponded to the described MRI abnormalities. In addition, the presence of lesional tissue was confirmed by an intraoperative consultation with Neuropathology.

\section{Pathology: Dr. Das}

The most striking neuropathologic finding on routine stains was that of a florid reactive and inflammatory process (Figure 2). This was dominated by perivascular and infiltrating CD3-positive T-lymphocytes, diffuse abundant activated human leukocyte antigen DR-positive microglia, and dramatic reactive astrogliosis. In subcortical white matter, patchy myelin loss was accompanied by another key finding. Many putative oligodendroglial nuclei were swollen by eosinophilic hyaline inclusions. Immunohistochemistry for polyomavirus (SV40) confirmed the nature of the intranuclear inclusions. Further inclusions were also found among astrocytes and select cortical neurons. The final diagnosis was progressive multifocal leukoencephalopathy (or John Cunningham virus [JCV] encephalitis).

\section{Patient Follow-Up: Dr. IKeda}

Before the diagnosis of progressive multifocal leukoencephalopathy (PML), the patient was treated with a five-day course of intravenous methylprednisolone and a prednisone taper with the question of central nervous system inflammatory disease or vasculitis. She also received one cycle of cyclophosphamide. During her hospitalization, she developed focal motor seizures involving the right arm that were controlled with phenytoin and clobazam. Once the diagnosis of PML was made, the immunosuppressive treatment was discontinued and she was started on mefloquine $500 \mathrm{mg}$ twice weekly and mirtazapine $30 \mathrm{mg}$ daily. This provided clinical stabilization over a one-year period, with mild improvement in strength. However, she remains wheelchairbound with occasional breakthrough seizures that are usually associated with infection. She is living in long-term care because of her motor dysfunction, and her cognition remains stable with repeat Montreal Cognitive Assessment testing demonstrating no change.

\section{DISCUSSION: DR. IKEDA}

PML is a central nervous system demyelinating disease caused by the polyoma JCV. JCV is ubiquitous and can be detected in blood samples of $50-90 \%$ of the population; however, PML typically develops in immunocompromised individuals. ${ }^{1}$ Initial infection with JCV is asymptomatic, and the virus is thought to gain entry to the body through infection of the tonsils. It then lays dormant in the kidneys, bone marrow, and lymphoid tissue. ${ }^{2}$ JCV gains access to cells through clathrin-mediated endocytosis using sialic acid and serotonin receptors., ${ }^{2,3}$ The presence of both receptor types may facilitate entry into host cells and may partially explain the limited trophism of the virus and preferential infection of neural tissue. ${ }^{2,3}$ After reactivation, which typically occurs with immunosuppression, JCV is thought to gain access to the brain through transmission in circulating B cells, which cross the blood-brain barrier and allow JCV access to neurons and glial cells. ${ }^{1,2}$ Oligodendrocytes are the most commonly affected neural cells; however, astrocytes and neurons may also be infected. ${ }^{4}$

Although PML most commonly occurs in immunocompromised individuals, it can be seen in people who are not immunocompromised. Gheuens et $\mathrm{al}^{5}$ reported data from 38 cases of PML in patients without a clear source of immunocompromise and found no underlying diagnosis in $57.9 \%$ of cases. In the remaining cases, hepatic failure, renal failure, pregnancy, and dementia were felt to have been the precipitating factor leading to the development of PML. Of the cases without another diagnosis, $22.7 \%$ were found to have an isolated CD4+ lymphopenia. ${ }^{5}$ The significance of a CD4 + lymphopenia is unclear because it is occasionally seen in association with PML, often as an incidental finding at the time of PML diagnosis. ${ }^{6}$

Diagnosis of PML is made with a combination of clinical features, radiographic findings, and histopathology or viral 

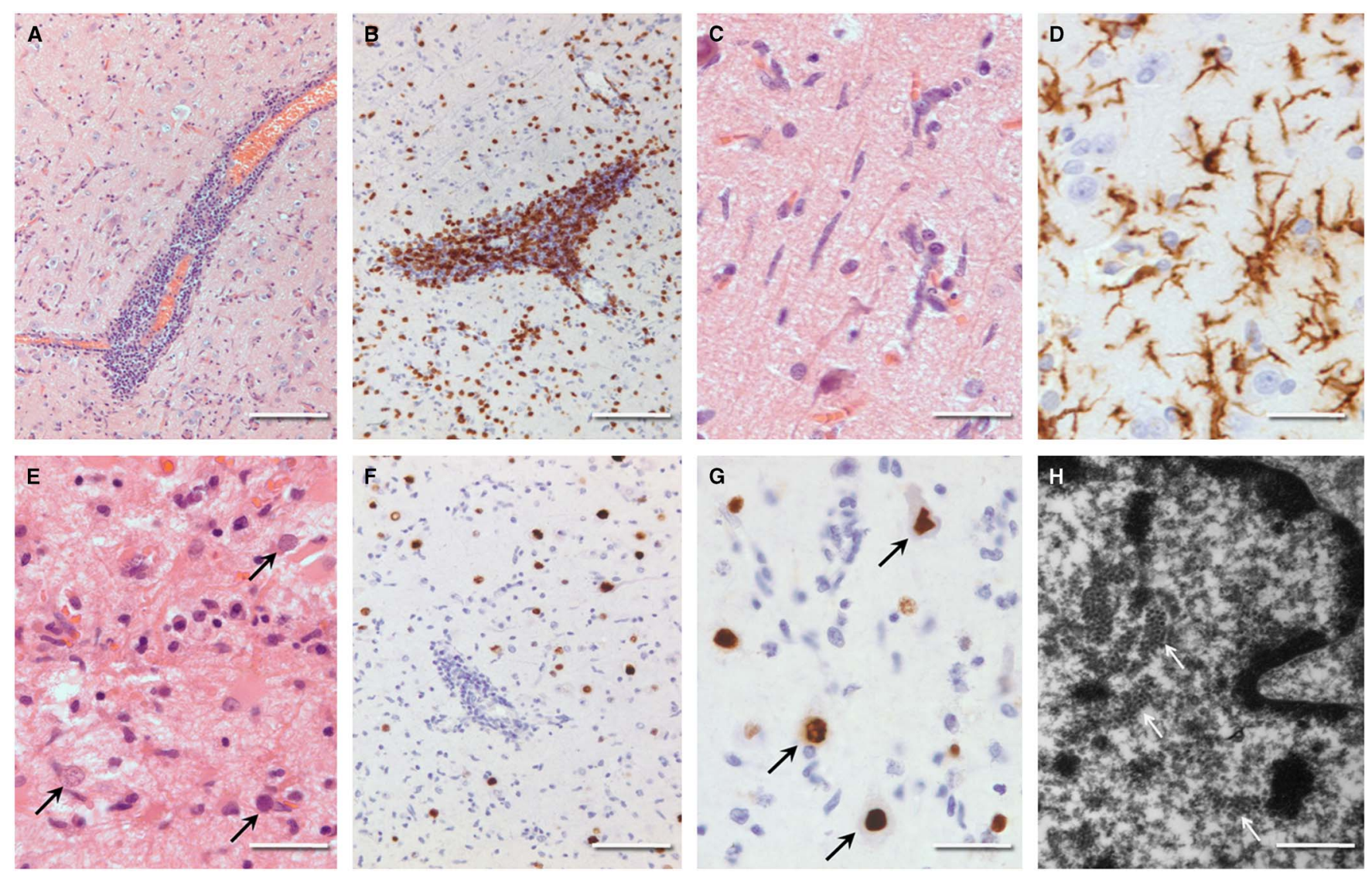

Figure 2: Pathology photomicrographs. (A) Densely gliotic parenchyma contains patchy intraparenchymal perivascular and intramural chronic inflammatory infiltrates (hematoxylin and eosin, bar $=100 \mu \mathrm{m})$. (B) The majority of perivascular/intramural infiltrates are $T$ cells $(C D 3$ immunoperoxidase, bar $=100 \mu \mathrm{m})$. (C) The cerebral cortex is diffusely decorated with rod-shaped nuclei of hyperplastic and activated microglia (hematoxylin and eosin, bar $=25 \mu \mathrm{m}$ ). (D) Confirmation of microglial activation with anti-human leukocyte antigen DR immunohistochemistry (anti-human leukocyte antigen DR immunoperoxidase, bar $=25 \mu \mathrm{m}$ ). (E) Hyaline/ground-glass eosinophilic inclusions (arrows) replace the normal chromatin pattern of many nuclei. This is particularly prominent among oligodendroglia in subcortical white matter (hematoxylin and eosin, bar $=25 \mu \mathrm{m}) .(F)$ The nuclear inclusions express SV40 polyoma antigens. Note the variable rectilinear profiles of the inclusions as influenced by the crystalline architecture of the viral capsid assemblies (anti-SV40 immunoperoxidase, bar $=50 \mu \mathrm{m}$ ). (G) Examples of neuronal infection (arrows) (anti-SV-40 immunoperoxidase, bar $=25 \mu \mathrm{m})$. (H) Ultrastructural confirmation of intranuclear viral capsid assemblies (arrows) averaging $40 \mathrm{~nm}$ in diameter $($ bar $=500 \mathrm{~nm})$.

polymerase chain reaction. ${ }^{7}$ The clinical course is typically subacute over weeks to months and commonly presents with hemiparesis, language difficulties, visual disturbances, or gait difficulties. Less commonly, PML can present with headaches, seizures, sensory loss, and cognitive decline. Radiological findings consistent with PML include T2 and fluid attenuation inversion recovery hyperintense lesions of the white matter with corresponding T1 hyperintensities, often involving the parietal and occipital lobes. ${ }^{1}$ Lesions are typically multifocal, but can be single. Contrast enhancement is uncommon and seen in only $15 \%$ of cases. ${ }^{7}$ CSF polymerase chain reaction for JCV can be confirmatory if positive, but if negative, histopathological diagnosis with a brain biopsy may be required. The classic histopathological triad consists of multifocal demyelination; hyperchromatic, enlarged oligodendroglial nuclei; and bizarre, giant astrocytes with hyperchromatic nuclei. ${ }^{7}$ Other supporting evidence includes immunohistochemistry for polyoma virus and electron microscopy demonstrating viral inclusions.

Prognosis of patients with PML is poor, with an overall one-year survival rate of $50 \%{ }^{8}$ Some features that may indicate an improved survival rate include gadolinium enhancement on MRI and detectable CD8 + cytotoxic T lymphocytes. These features may indicate reconstitution of the immune system, or the immune reconstitution inflammatory syndrome that can assist in fighting the JCV infection. ${ }^{9}$

There is no standard treatment for PML; however, the goal remains reconstitution of the immune system. ${ }^{1,8}$ In those without a source of immunocompromise, this is difficult. Mefloquine, an antimalarial agent, was found in vitro to inhibit JCV infection and replication; however, a randomized controlled trial of mefloquine for PML was negative. ${ }^{10}$ Other agents with negative clinical trials include a randomized controlled trial with cytarabine ${ }^{11}$ and an open-label study with cidofovir. ${ }^{12}$ However, there have been many case reports describing these treatments or combinations of treatments to be effective. Mirtazapine has gained interest in treating PML lately because it is a 5HT2a receptor antagonist and could theoretically block entry of JCV via the serotonin receptors. ${ }^{8}$ There are case reports of mirtazapine improving outcomes, often in combination with mefloquine, but no randomized study has been completed. In those with an element of inflammation, or 
immune reconstitution inflammatory syndrome, treatment with steroids has been proposed, but remains controversial because it involves further suppressing the immune system. ${ }^{8}$

\section{Conclusion}

This case demonstrates pathologically proven PML in an individual with an isolated lymphopenia. The lymphopenia was felt to be of uncertain significance. She was otherwise immunocompetent. Treatment with intravenous corticosteroids followed by the combination of mefloquine and mirtazapine provided clinical stabilization and slight improvement. The diagnosis of PML can be made in immunocompetent individuals and requires a high index of suspicion in combination with an appropriate clinical history of subacute progressive neurological symptoms and radiographic findings. Definitive diagnosis requires the demonstration of JCV in CSF or the requisite histopathological findings on biopsy. Although the prognosis is uniformly poor, treatment with varying agents may provide some clinical stabilization, as did treatment with mefloquine and mirtazapine in the present case.

\section{Disclosures}

The authors declare that they have no conflicts of interest.

\section{Statement of Authorship}

KMI drafted and revised the article. RH revised the article, provided contextual guidance and the pathology images. MS provided the discussion. AL provided the radiological description and radiological images. DS provided the surgical descriptions. SD provided the pathological description. SMM edited the article.

\section{Consent}

Written informed consent was obtained from the patient for publication of this Case Report and accompanying images. A copy of the written consent is available for review by the Editor-in-Chief of this journal.

\section{REFERENCES}

1. Brew BJ, Davies NW, Cinque P, Clifford DB, Nath A. Progressive multifocal leukoencephalopathy and other forms of JC virus disease. Nat Rev Neurol. 2010;6:667-79.

2. Ferenczy MW, Marshall LJ, Nelson CD, Atwood WJ, Nath A, Khalili K, et al. Molecular biology, epidemiology, and pathogenesis of progressive multifocal leukoencephalopathy, the JC virus-induced demyelinating disease of the human brain. Clin Microbiol Rev. 2012;25:471-506.

3. Elphick GF, Querbes W, Jordan JA, Gee GV, Eash S, Manley K, et al. The human polyomavirus, JCV, uses serotonin receptors to infect cells. Science. 2004;306:1380-3.

4. Gheuens S, Wuthrich C, Koralnik IJ. Progressive multifocal leukoencephalopathy: why gray and white matter. Annu Rev Pathol. 2013;8:189-215.

5. Gheuens S, Pierone G, Peeters P, Koralnik IJ. Progressive multifocal leukoencephalopathy in individuals with minimal or occult immunosuppression. J Neurol Neurosurg Psychiatry. 2010;81:247-54.

6. Delgado-Alvarado M, Sedano MJ, Gonzalez-Quintanilla V, de Lucas EM, Polo JM, Berciano J. Progressive multifocal leukoencephalopathy and idiopathic CD4 lymphocytopenia. J Neurol Sci.. 2013;327:75-9.

7. Berger JR, Aksamit AJ, Clifford DB, Davis L, Koralnik IJ, Sejvar JJ, et al. PML diagnostic criteria: consensus statement from the AAN neuroinfectious disease section. Neurology. 2013;80: 1430-1438.

8. Tan CS, Koralnik IJ. Progressive multifocal leukoencephalopathy and other disorders caused by JC virus: clinical features and pathogenesis. Lancet Neurol. 2010;9:425-37.

9. Marzocchetti A, Tompkins T, Clifford DB, Gandhi RT, Kesari S, Berger JR, et al. Determinants of survival in progressive multifocal leukoencephalopathy. Neurology. 2009;73:1551-8.

10. Clifford DB, Nath A, Cinque P, Brew BJ, Zivadinov R, Gorelik L, et al. A study of mefloquine treatment for progressive multifocal leukoencephalopathy: results and exploration of predictors of PML outcomes. J Neurovirol. 2013;19:351-8.

11. Hall CD, Dafni U, Simpson D, Clifford D, Wetherill PE, Cohen B, et al. Failure of cytarabine in progressive multifocal leukoencephalopathy associated with human immunodeficiency virus infection. AIDS clinical trials group 243 team. N Engl J Med. 1998:338:1345-51.

12. Marra CM, Rajicic N, Barker DE, Cohen BA, Clifford D, Donovan Post MJ, et al. A pilot study of cidofovir for progressive multifocal leukoencephalopathy in AIDS. AIDS. 2002;16:1791-7. 